\title{
Lipomesosalpinx: a rare possible missed tubal factor of infertility
}

\author{
Atef Darwish \\ Received: 27 May 2013 / Accepted: 30 October 2013 / Published online: 12 November 2013 \\ (C) Springer-Verlag Berlin Heidelberg 2013
}

\begin{abstract}
This study aims to estimate the proportion of significant mesosalpingeal adipose tissue condensation (lipomesosalpinx, at least of a caliber similar to the ampulla of the ipsilateral tube regardless of well-defined or poorly defined margins) among infertile women subjected to diagnostic laparoscopy. This study is a cross-sectional study set at a specialized endoscopic center. All infertile women scheduled for diagnostic/therapeutic laparoscopy during the period between July 1994 and December 2012 was included in this study. Interventions used were preoperative hysterosalpingography, transvaginal ultrasonography, as well as body mass index for all cases. Laparoscopic documentation of a significant mesosalpingeal condensation of adipose tissue as well as histopathologic assessment of the adipose tissues in some cases was observed. The main outcome measures included number of cases with unilateral or bilateral lipomesosalpinx. Significant lipomesosalpinx was diagnosed in 145 (5.7\%) out of 2,563 cases examined by laparoscopy. In all but seven cases, lipomesosalpinx was seen bilaterally $(99.7 \%)$. There was insignificant correlation between those cases and high body mass index when compared to the rest of the cases. Infertility was unexplained by laparoscopy in 621 cases (24.3\%) while laparoscopy diagnosed etiologic factors in 1, $942(75.7 \%)$ cases. Lipomesosalpinx was seen in 46 (7.4\%) and $79(3.9 \%)$ of the unexplained and explained cases, respectively, without a statistically significant difference $(P=$ 0.48). Despite being a rare laparoscopic finding, significant lipomesosalpinx should be reported and documented as a possible missed tubal factor of infertility. Whether to treat
\end{abstract}

A. Darwish

Woman's Health University Center, Assiut University, Assiut, Egypt

A. Darwish $(\bowtie)$

Department of Obstetrics \& Gynecology, Woman's Health

University Hospital, 71111 AssiutP.O. Box: (1)Assiut, Egypt

e-mail: atef_darwish@yahoo.com lipomesosalpinx or not, bilaterally or unilaterally and by which means, require further studies with proper secondlook laparoscopy.

Keywords Lipomesosalpnix · Laparoscopy $\cdot$ Infertility · Tubal factor

\section{Introduction}

There is a general consensus among gynecologists that tubal patency at hysterosalpingography (HSG) is quite assuring about tubal factor and they proceed to investigate other factors or advise patients to try assisted reproduction. Actually, the fallopian tube is a complex paired organ, not a simple tubing. The classic tubal factors include post-inflammatory peritubal adhesions and prominal or diatal tubal occlusion [1] which can be easily diagnosed by most gynecologists based on HSG. Other rare tubal diseases are seldom investigated. For instance, salpingitis isthmica nodosa which is a nodular swelling of the isthmic segment of the fallopian tube are rarely reported [2]. Anatomically, mesosalpinx is defined as the part of the broad ligament enclosing a fallopian tube forming its mesentery. Histologically, it is formed of a thin layer of squamous epithelium and a small amount of loose areolar connective tissue [3, 4]. It contains sympathetic ganglia and plexuses [5]. Laparoscopically, mesosalpinx is a thin vascular layer without evident fat in most cases.

Unexplained infertility is a real challenge for gynecologists. It is idiopathic in the sense that its cause remains unknown even after an infertility work-up, usually including semen analysis in the man and assessment of ovulation and fallopian tubes in the woman [6]. In Assiut, we believe that diagnostic laparoscopy is an integral step of the diagnostic work-up of any infertile couple before saying the term "unexplained". With time, interest to discover minute lesions that may affect fertility 
increased at our institution [7]. In practice, we observe some fatty tissue condensation in the mesosalpinx in some cases that deserve studying why it is present in some women. To make this study valuable, we considered mesosalpingeal adipose tissue significant if its caliber was at least similar or exceeds the caliber of the ampulla of the ipsilateral fallopian tube regardless of the appearance of its borders. The tested hypothesis is a significant lipomesosalpinx that would hinder tubal motility and would be a cause of infertility. This study aims to estimate the proportion of significant mesosalpingeal adipose tissue condensation (lipomesosalpinx) among infertile women subjected to diagnostic/operative laparoscopy.

\section{Patients and methods}

This study was conducted between July 1996 and December 2012 at the Endoscopic Unit of the Woman's Health University Hospital, Assiut University, Egypt. It was approved by the institutional review board (IRB) of the Faculty of Medicine. All patients gave a clear written consent to participate in this study. It prospectively comprised 2,563 infertile patients submitted to video-assisted laparoscopy for diagnostic or operative purposes (Table 1). Preoperative metric body mass index (BMI) estimation and transvaginal ultrasonography (TVS) were done as a routine for all of the cases. Metric BMI is calculated as weight in kilograms divided by height in meters squared. Meticulous evaluation of HSG was made to identify tubal shape and patency whenever available. At laparoscopy, a thorough visualization of the mesosalpinx for evidence of adipose tissue condensation was reported. We considered mesosalpingeal adipose tissue significant if its caliber was at least similar or exceeds the caliber of the ampulla of the ipsilateral fallopian tube (Fig. 1). In all cases, using tubal chromopertubation, tubal patency was assessed and the relationship of the mass to the tubal lumen was recorded. Moreover, observation of the range of mobility of the tubes to the Douglas pouch was reported.

Table 1 Laparoscopic procedure for 2,563 cases

\begin{tabular}{lc}
\hline Laparoscopic finding & No (\%) \\
\hline Diagnostic (normal findings) & $621(24.3)$ \\
Operative (abnormal findings) & $1942(75.7)$ \\
Ovarian drilling for polycystic ovaries & $523(20.4)$ \\
Salpingectomy for hydrosalpinx & $165(6.4)$ \\
Oophorectomy for a benign ovarian mass & $46(1.7)$ \\
Adhesiolysis & $344(13.4)$ \\
Cystectomy of ovarian cyst & $298(11.6)$ \\
Myomectomy & $29(1.1)$ \\
Miscellaneous & $587(22.9)$ \\
\hline
\end{tabular}

More one than procedure was required in 50 cases

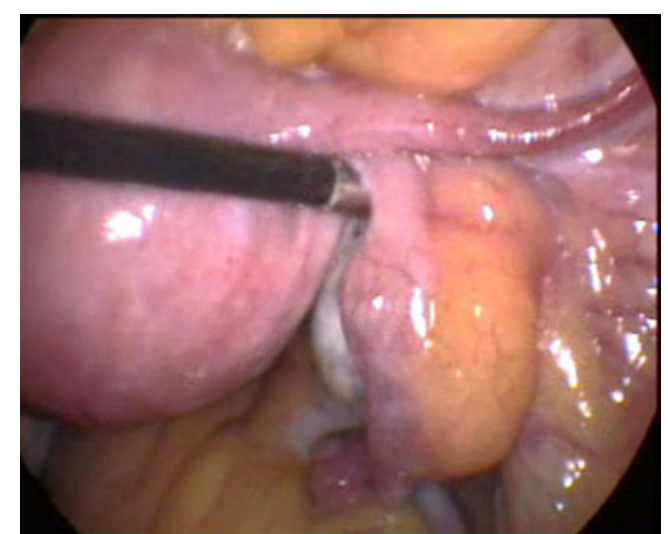

Fig. 1 Laparoscopic appearance of lipomesosalpinx

Based on the recommendations of the IRB, only unilateral excision or lysis of significant lipomesosalpinx was performed even if it was seen bilaterally in an otherwise normal genital tract anatomy at laparoscopy. Since it is a preliminary study, ethics recommended if any intervention should be unilateral until clear results of better bilateral excision of lipomesosalpinx with second-look laparoscopies. The side of the tube that will be operated upon was recorded. If this fatty condensation is pedunculated, it was excised with a bipolar scissors. If nonpedunculated but localized, a small microsurgical incision of the mesosalpinx was made followed by extraction of the adipose tissue with a fine-grasping forceps. The mesosalpingeal defect was then coagulated with bipolar forceps. Sutures or monopolar diathermy were not used in any case to minimize the risk of peritubal adhesions or tubal damage. On the other hand, if the adipose tissue mass was diffuse and ill-defined, it was coagulated as far as possible from the fallopian tube utilizing a 3-mm bipolar needle till complete melting of the adipose tissue. In most cases, histopathologic examination of the adipose tissue biopsy (Fig. 2) was done.

Copious peritoneal washing was done followed by leaving about $1 \mathrm{~L}$ of lactated Ringer's solution intraperitoneally. All the laparoscopic findings were correlated with the preoperative TVS findings. The $X^{2}$ test was used to compare the studied groups. For each comparison, $P>0.05$ was considered not significant, while $P \leq 0.05$ was considered significant.

\section{Results}

This study included 2,563 infertile patients submitted to diagnostic or operative laparoscopy (Table 1). They were in the child-bearing period with a mean age of 24.5 years and mean parity of 1 . Preoperatively, TVS was done for all cases that failed to detect any paraovarian echogenic condensation in all cases. Body mass index was calculated for all cases. Its mean was 29.4 with statistically insignificant correlation to lipomesossalpinx $(P=0.12)$. Significant lipomesosalpinx 


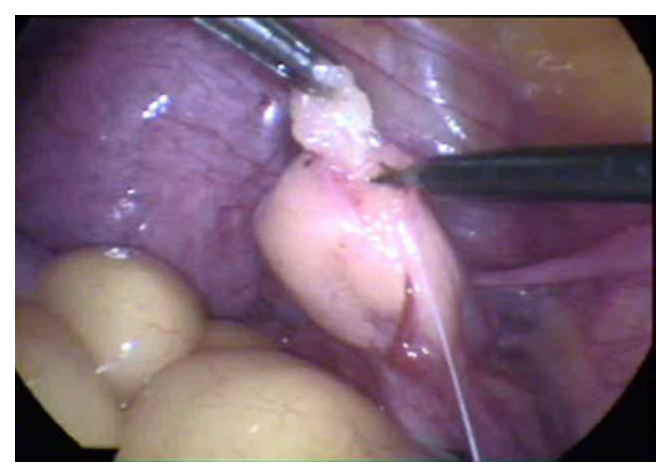

Fig. 2 Biopsy taking of lipomesosalpinx

was diagnosed in 145 cases (5.7 \%). In all but seven cases, lipomesosalpinx was diagnosed bilaterally $(99.7 \%)$. Infertility was unexplained by laparoscopy in 621 cases $(24.3 \%)$ while the cause of infertility could be explained in 1,942 (75.7\%) of the cases. Lipomesosalpinx was diagnosed in 46 cases $(7.4 \%)$ and $79(3.9 \%)$ in both groups respectively without statistically significant difference $(P=0.48)$. Biopsy of lipomesosalpinx revealed a normal adipose tissue in all cases. Surgical management of lipomesosalpinx was done, but data were excluded according to the aim of this study.

\section{Discussion}

Tubal factor infertility accounts for nearly one quarter of all cases of infertility [8]. The fallopian tubes may be abnormal in structure or function. Structural disorders can block the fallopian tubes. They include tubal scarring or blockage most commonly from pelvic infections, prior abdominal surgeries, and endometriosis. Practically, many gynecologists are reluctant when reporting on diagnostic laparoscopy. Some perform a single-puncture intraumbilical procedure that neglects an auxiliary portal for proper grasping of the adnexa and thorough evaluation of the ovarian fossa. Most of them comment on tubal patency only and neglect tubal morphology, size, length, and proximity to the pouch of Douglas. Rarely, they comment on the mesosalpinx and Wolffian duct remnants. This study directs attention towards more concentration on some factors that would affect tubal motility and commonly missed by gynecologists. Previously, some authors reported on hydatid of Morgagni as a cause of unexplained infertility (UI) [9]. Likewise, an old study diagnosed fimbrial agglutinations $(25 \%)$, accessory tubes (13\%), accessory ostia (10\%), phimoses (13\%), and sacculations (7\%) more in the infertile women [10]. Tubal abnormalities would affect the prognosis of natural pregnancy as well as laparoscopic gamete intrafallopian transfer [11]. At our institution, we consider tubal sacculations, diverticulae, convolutions, phimosis, or fimbrial agglutination as laparoscopic criteria of subtle tubal endometriosis specially if seen with other typical or atypical endometriotic tubal or peritoneal lesions. Proper endoscopic training would eliminate all these mistakes that would affect diagnosis as well as therapy.

Mesosalpingeal lesions include paratubal cyst [7], leiomyosarcoma of the broad ligament [12], choristoma of the heterotopic adrenal tissue [13], primary fallopian tube carcinoma [14], or lipoma of the broad ligament [15]. Preciously, among 1,853 cases subjected to laparoscopy, we succeeded to diagnose a paratubal or paraovarian cyst in 118 patients $(15.7 \%)$ [7]. Fat condensation in the mesosalpinx is not described in textbooks on histology, pathology, or even endoscopic surgery as far as I know. Due to our interest in missed factors of infertility, we tried to study the clinical significance of lipomesosalpinx. To be practical, we excluded cases with small amounts of adipose tissue that would not be expected to affect tubal motility. In this study, preoperative TVS failed to diagnose lipomesosalpinx in all cases. Fallopian tubes are not usually visualized on a routine transvaginal sonographic examination unless outlined by fluid. However, the interstitial segment may be identified on TVS as an echogenic line arising from the endometrial canal and extending through the uterine wall. When surrounded by intraperitoneal fluid, the remaining segments of the fallopian tubes are commonly seen as tubular structures extending between the uterus and the ovaries. Fallopian tubes are best visualized on sonography when thickened or fluid-filled as a result of pelvic inflammatory disease, torsion, ectopic pregnancy, or tumors [15]. Nevertheless, we still recommend performing TVS routinely prior to laparoscopy to detect important findings like paraovarian cysts [7] and more importantly intrauterine lesions that would make concomitant hysteroscopy a mandatory step.

Despite similarity of the histopathologic appearance of lipomesosalpinx to any adipose tissue in the body, failure to prove any correlation between lipomesosalpinx and obesity would support screening for lipomesosalpinx in all infertile women.

To date, there is no uniform definition for UI [6]. With the marvelous advancement in illumination and magnification, endoscopy would add a lot for the diagnostic work-up for cases with UI. The findings of this and our previous [7] studies would support the central role of dual endoscopy (combined laparoscopy and hysteroscopy) in all cases of infertility despite not being clearly stated by most of the infertility-interested societies when defining UI. One of the promising and attractive options for evaluation of subtle tubal and mesosalpingeal lesions is hydrolaparoscopy which offers a comparable accuracy to laparoscopy in $96.1 \%$ of cases [16].

Despite being described for a long time [17], the impact of fatty condensation of the mesosalpinx on fertility is not yet 
studied so far; this is the first study in English literature to address this point and to report it in $5.7 \%$ of infertile women. Lipomesosalpinx would theoretically affect tubal motility and more importantly leads to failure to reach the pouch of Douglas for ovum pick up despite being a patent tube. Nevertheless, the results of this study failed to prove a positive correlation between lipomesosalpinx and unexplained infertility. This calls for a larger sample-sized multicentric study. The main value of this study is to direct attention to mesosalpingeal lesions that would affect fertility. Despite being a rare laparoscopic finding, lipomesosalpinx should be reported and documented as a missed tubal factor of infertility. Whether to treat lipomesosalpinx or not, bilaterally or unilaterally and by which means, require further studies with proper secondlook laparoscopy.

Informed consent All procedures followed were in accordance with the ethical standards of the Responsible Committee on Human Experimentation (institutional and national) and with the Helsinki Declaration of 1975 as revised in 2000 (5). Informed consent was obtained from all patients for being included in the study.

\section{References}

1. Gomel V, Taylor PJ, Yuzpe A, Roux J (1986) Laparoscopy and hysteroscopy in gynecologic practice. Year Book, Chicago

2. Chawla N, Kudesia S, Azad S, Singhal M, Rai SM (2009) Salpingitis isthmica nodosa. Indian J Pathol Microbiol 52(3):434-435
3. Kessel R (1998) Medical histology. Oxford University Press, London, $\mathrm{p} 486$

4. Gartner LP, Hiatt JL (2007) Color textbook of histology, 3rd edn. Elsevier, New York, pp 477-78

5. Weiss L, Greep RO (1977) Histology, 4th edn. McGraw-Hill, New York, $\mathrm{p} 920$

6. Ray A, Shah A, Gudi A, Homburg R (2012) Unexplained infertility: an update and review of practice. Reprod Biomed Online 24(6):591602

7. Darwish AM, Amin AM, Mohammad SA (2003) Laparoscopic management of paratubal and paraovarian cysts. J Soc Laparoendosc Surg 7(2):101-106

8. Child T (2013) Optimising the management of patients with infertility. Practitioner 257(1759):19-22, 2-3

9. Rasheed SM, Abdelmonem AM (2011) Hydatid of Morgagni: a possible underestimated cause of unexplained infertility. J Obstet Gynecol Reprod Biol 158(1):62-66

10. Yablonski M, Sarge T, Wild RA (1990) Subtle variations in tubal anatomy in infertile women. Fertil Steril 54(3):455-458

11. Fakih H, Marshall J (1994) Subtle tubal abnormalities adversely affect gamete intrafallopian transfer outcome in women with endometriosis. Fertil Steril 62(4):799-801

12. Kolusari A, Ugurluer G, Kosem M, Kurdoglu M, Yildizhan R, Adali E (2009) Leiomyosarcoma of the broad ligament: a case report and review of the literature. Eur J Gynaecol Oncol 30(3):332-334

13. Janovski NA (1966) Choristoma of heterotopic adrenal tissue in mesosalpinx. Obstet Gynecol 28(3):380-382

14. Huang WC, Yang SH, Yang JM (2005) Ultrasonographic manifestations of fallopian tube carcinoma in the fimbriated end. J Ultrasound Med 24(8):1157-1160, quiz 1161-2

15. Benjaminov O, Atri M (2004) Sonography of the abnormal fallopian tube. AJR Am J Roentgenol 183(3):737-742

16. Ezedinma NA, Phelps JY (2012) Transvaginal hydrolaparoscopy. JSLS 16(3):461-465

17. Lockyer C (1919) Lipoma of the broad ligament. Proc R Soc Med 12(Obstet Gynaecol Sect):195-199 